\title{
On the effect of protein conformation diversity in discriminating among neutral and disease related single amino acid substitutions
}

\author{
Ezequiel Juritz ${ }^{1}$, Maria Silvina Fornasari ${ }^{1}$, Pier Luigi Martelli ${ }^{2}$, Piero Fariselli ${ }^{3}$, Rita Casadio ${ }^{2}$, Gustavo Parisi ${ }^{1 *}$ \\ From SNP-SIG 2011: Identification and annotation of SNPs in the context of structure, function and disease \\ Vienna, Austria. 15 July 2011
}

\begin{abstract}
Background: Non-synonymous coding SNPs (nsSNPs) that are associated to disease can also be related with alterations in protein stability. Computational methods are available to predict the effect of single amino acid substitutions (SASs) on protein stability based on a single folded structure. However, the native state of a protein is not unique and it is better represented by the ensemble of its conformers in dynamic equilibrium. The maintenance of the ensemble is essential for protein function. In this work we investigated how protein conformational diversity can affect the discrimination of neutral and disease related SASs based on protein stability estimations. For this purpose, we used 119 proteins with 803 associated SASs, 60\% of which are disease related. Each protein was associated with its corresponding set of available conformers as found in the Protein Conformational Database (PCDB). Our dataset contains proteins with different extensions of conformational diversity summing up a total number of 1023 conformers.

Results: The existence of different conformers for a given protein introduces great variability in the estimation of the protein stability $(\triangle \triangle G)$ after a single amino acid substitution (SAS) as computed with FoldX. Indeed, in 35\% of our protein set at least one SAS can be described as stabilizing, destabilizing or neutral when a cutoff value of $\pm 2 \mathrm{kcal} / \mathrm{mol}$ is adopted for discriminating neutral from perturbing SASs. However, when the $\triangle \Delta G$ variability among conformers is taken into account, the correlation among the perturbation of protein stability and the corresponding disease or neutral phenotype increases as compared with the same analysis on single protein structures. At the conformer level, we also found that the different conformers correlate in a different way to the corresponding phenotype.
\end{abstract}

Conclusions: Our results suggest that the consideration of conformational diversity can improve the discrimination of neutral and disease related protein SASs based on the evaluation of the corresponding Gibbs free energy change.

\section{Background}

Human single nucleotide polymorphisms (SNPs) are the most frequent type of genetic variation in humans. Less than $1 \%$ variations are associated with non-synonymous coding SNPs (nsSNPs). About 64,971 nsSNPs are presently listed as human polymorphisms and disease single amino acid substitutions, SASs, (http://www.uniprot.org/

\footnotetext{
* Correspondence: gusparisi@gmail.com

'Departamento de Ciencia y Tecnología, Universidad Nacional de Quilmes, Buenos Aires, Argentina

Full list of author information is available at the end of the article
}

docs/humsavar) and approximately $40 \%$ of these SASs are disease related.

It has been documented that in proteins a single amino acid substitution (SAS), can produce the loss of function in different ways. Although the less frequently found [1], the most obvious mechanism at the disease origin is due to change of key residues participating directly in protein function. This is the case when residue substitution occurs at the active site or in binding-sites for substrate and/or allosteric regulators [2-4]. When the biological functional unit is a complex, SASs at the subunit interface may also 
hamper the activity $[4,5]$. A second possible mechanism is related with the perturbation of protein stability. Residue substitution can indeed destabilize the native protein fold $[1,6]$. Also stabilizing residue changes have been reported to be associated with diseases $[7,8]$. Furthermore, related with protein stability alteration, the origin of pathogenesis was also related with anomalous post-translational modifications [9] and aggregation [10]. The correlation among protein SASs and their involvement in human diseases has been proven to be moderate [11], suggesting that change in protein stability is not the only source of diseases.

Protein stability can be estimated measuring the variation of Gibbs free energy $(\Delta \Delta G)$ between the folded and unfolded state of the protein. Most of the experimental data reported in literature are contained in ProTherm [12], a thermodynamic database of proteins and their variation in different organisms. Alternatively, several computational methods have been developed to estimate stability changes caused by substitution of lateral side chains in proteins $\left(\Delta \Delta G=\Delta G_{\text {wild }}-\Delta G_{\text {mutated }}\right)$. Most of them rely on the analysis of the energetic and/or structural perturbation introduced by the variations in the protein native structure. Although computationally intensive, early methods used all atom models to estimate $\Delta \Delta G$ [13]. Soon later, simplified potentials coupled with limited conformational searches [14,15] and the use of different types of potentials, like those based on hydrophobic interactions [16], secondary structure [17], interresidue contacts [18] and knowledge-based [19], allowed to study the effect of different mutations in proteins in a reasonable computational time. Recently machine learning based approaches have been implemented for the prediction of $\Delta \Delta G$ in proteins upon residue substitution taking as input either the protein structure or sequence (for a recent review see [20]). The discrimination among disease related and neutral SASs can be investigated by determining $\Delta \Delta G$ upon residue change in the protein. This analysis is based on the notion that most harmful SASs are related to protein stability perturbation above a certain threshold $\Delta \Delta \mathrm{G}( \pm 1 \mathrm{kcal} / \mathrm{mol})$. Most methods suited to predict free energy changes have been recently benchmarked in relation to their ability to discriminate disease from neutral SASs based on the corresponding $\Delta \Delta G$ value and their performance has been proven to be rather poor [20].

In most methods, predictions of SASs effects are commonly estimated using a single structure of the corresponding protein [21-24]. This approach apparently underestimates the well established concept that the native state of a protein is better represented by an ensemble of conformers [25-27]. The conformational ensemble is a key concept to explain essential properties of proteins like function [28-30], enzyme and antibody promiscuity [31,32], enzyme catalytic power [33], signal transduction
[34], protein-protein recognition [35] and the origin of new functions [36]. Conformers describing the native state of a protein exist in a dynamic equilibrium which changes in response to the presence of ligands such as substrate or allosteric modulators that shift the relative conformational population $[37,38]$. From a practical perspective, conformational diversity could be described using experimentally available structures of the same protein obtained in alternative conditions. As these different structures for the same protein have been obtained under different conditions (for example presence of substrate, inhibitors or allosteric activators) they can be taken as snapshots of protein dynamics and then characterize putative conformers belonging to the native ensemble $[39,40]$. In this way, the description of the native ensemble of the protein will be more or less complete depending on available experimental data. A way to describe the extension of conformational diversity could be the estimation of the maximum RMSD measured between the available conformers. The distribution of the structural diversity extension measured in this way in the protein space was recently studied [41]. The analysis involves an all vs. all comparisons between structures of the same sequence deposited in PDB database [42]. Conformer distribution exhibits a peak at $0.3 \AA$ RMSD with a large skew that ends at about $24 \AA$ RMSD.

In the present work we investigated how the presence and extension of conformational diversity affect the estimation of $\Delta \Delta G$ in proteins with neutral and disease related SASs. Like protein function relies on the existence and preservation of the dynamic ensemble of conformers, the study of the effect of a SAS in each conformer of a given protein could help to understand the loss of function. We used a set of 803 SASs (482 disease related and 323 neutral) in 119 proteins showing different extension of conformational diversity. These proteins were taken from the Protein Conformational Database (PCDB) [43] a redundant collection of protein structures linked with biological information. $\Delta \Delta \mathrm{G}$ for each SAS in each conformer for a given protein was estimated using FoldX [44]. We found that the $\Delta \Delta G$ estimated value for a SAS highly depends on the conformer used in the estimation. In $35 \%$ and $58 \%$ of the studied proteins we found that at least one SAS could be classified as neutral, stabilizing or destabilizing depending on a $\Delta \Delta \mathrm{G}$ threshold value of $\pm 2 \mathrm{Kcal} / \mathrm{mol}$ and $\pm 1 \mathrm{Kcal} / \mathrm{mol}$ respectively. We also found that the consideration of conformational diversity increases the performance of the prediction of disease related SASs based on $\Delta \Delta G$ analysis. Our results show that the different conformers correlate in different ways with the phenotype (disease or neutral) and that, in most cases, one conformer per protein correlates perfectly with the corresponding phenotype. Our results indicate that the use of conformational 
diversity may be important to understand the effects of neutral and disease related SASs on protein stability.

\section{Results}

\section{Extension of protein conformational diversity}

The 119 proteins studied in this work were linked to the PCDB database [43]. All conformer coordinates contained in PCDB for each protein were derived from the PDB database (http://www.rcsb.org). The structures were obtained under different conditions, mainly in the presence of different ligands that shift the population equilibrium of the different conformers in the ensemble $[37,45]$. Our dataset has an average maximum root mean squared deviation (RMSD) between conformers of $2.51 \AA$ and an average number of conformers per protein of 8.6. The distribution of the maximum RMSD (the maximum RMSD displayed between all conformers of a given protein) is shown in Figure 1. Considering that the average RMSD for a protein crystallized under the same condition ranges from 0.1 and $0.4 \AA$ [42] and from the distribution shown in Figure 1, we concluded that our dataset contains proteins with moderated to extreme conformational diversity (for details on conformational diversity per protein see additional file 1). We also computed the relative accessible surface area (ASA) of the positions involved in SASs (neutral and disease related) as described in Methods. We found changes in the maximum $\Delta(\mathrm{ASA})$ between conformers, with a maximum value of $98.6 \AA^{2}$ and an average value of $12.0 \AA^{2}$ (Figure 2). This distribution reflects the structural changes at the SAS positions between conformers. In fact, using $\triangle$ (ASA) values, $33 \%$ of the proteins have at least one position that, depending on the chosen conformer, can be classified as buried $\left(\mathrm{ASA}<20 \AA^{2}\right)$ or solvent exposed $\left(\mathrm{ASA}>20 \AA^{2}\right)$. Previous observations suggested that $\Delta \Delta \mathrm{G}$ values upon residue substitution inversely

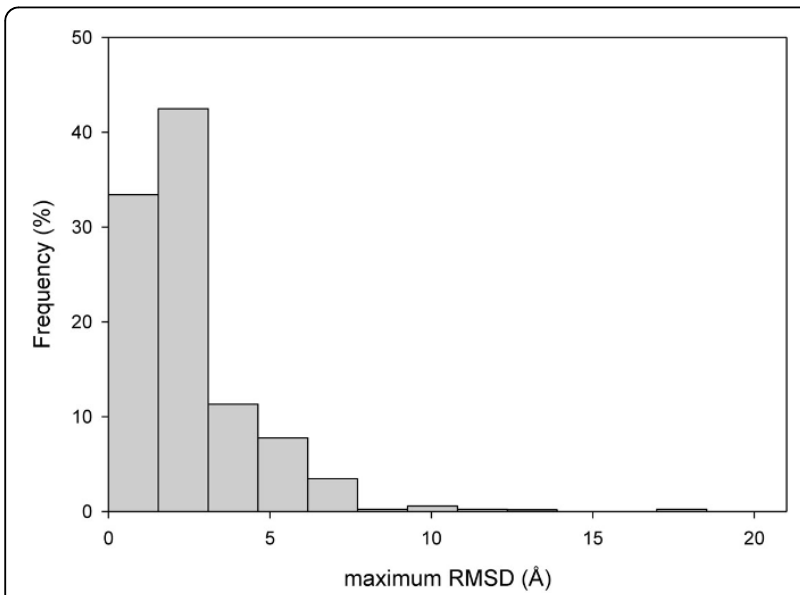

Figure 1 Distribution of maximum RMSD between conformers corresponding to the 119 proteins in the dataset used in this study.

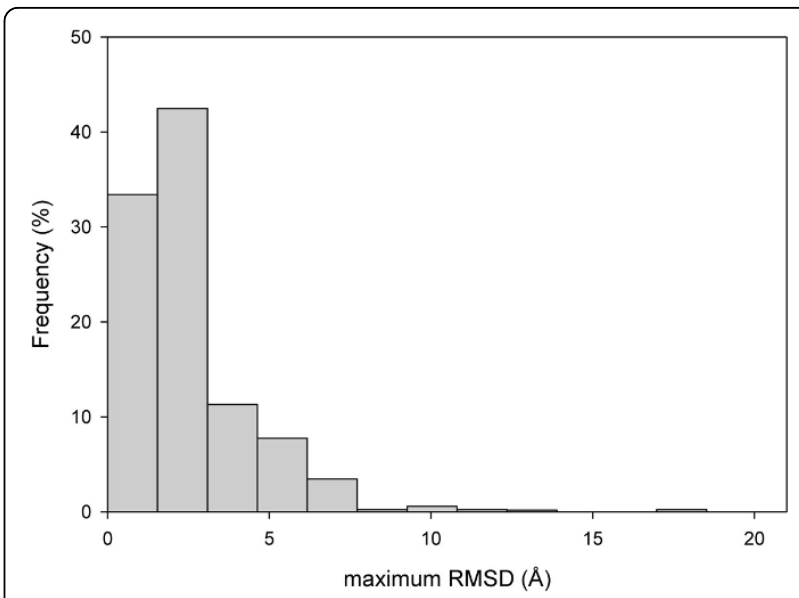

Figure 2 Distribution of $\triangle A S A\left(\AA^{2}\right)$ for substituted positions derived from the analysis of the conformational ensemble for each of the 119 proteins in the dataset.

correlate with the corresponding ASA values [46]. We therefore can expect large variations in the $\Delta \Delta G$ estimation upon changes on the different conformers considering the large ASA variation.

\section{Variation of $\Delta \Delta \mathrm{G}$ estimation using conformational diversity}

For each of the 803 SASs a $\Delta \Delta G$ estimation was performed for all the conformers of each protein using FoldX [44]. The accuracy of FoldX to predict stability changes has been discussed before $[20,46]$. For each mutation we registered the maximum and minimum $\Delta \Delta \mathrm{G}$ values and the maximum difference of the $\Delta \Delta G$ values among different conformers of the same protein (maximum $\Delta(\Delta \Delta \mathrm{G})$ ). The distributions of maximum and minimum $\Delta \Delta \mathrm{G}$ values and the distribution of the maximum difference of $\Delta \Delta G$ values (max. $\Delta(\Delta \Delta G)$ ) are shown in Figures 3 and 4 , respectively, for both disease related and neutral SASs. We found that maximum and minimum values of $\Delta \Delta \mathrm{G}$ of disease related SASs have higher (destabilizing) values compared with those of neutral SASs. The distributions of minimum $\Delta \Delta \mathrm{G}$ values have average values of $1.47 \mathrm{kcal} / \mathrm{mol}$ and of $0.38 \mathrm{kcal} / \mathrm{mol}$ for disease related and neutral SASs, respectively. This distribution difference is significant (Kolmogorov-Smirnov test with P-value $<110^{-5}$ ). In turn, average maximum $\Delta \Delta G$ values for disease and neutral SASs are 4.63 and $1.86 \mathrm{kcal} / \mathrm{mol}$ respectively (Kolmogorov-Smirnov test with $\mathrm{P}$-value $<110^{-5}$ ). Considering the distributions of maximum variation of the $\Delta(\Delta \Delta G)$, most of the values $(69 \%)$ are below $1 \mathrm{kcal} / \mathrm{mol}$. This value can be regarded as a typical standard error in the estimation of $\Delta \Delta \mathrm{G}$ [44] (Figure 4). However, $31 \%$ of the SASs have maximum $\Delta(\Delta \Delta \mathrm{G})$ above the standard error and a significant difference between the $\Delta \Delta G$ estimations of the different conformers. 

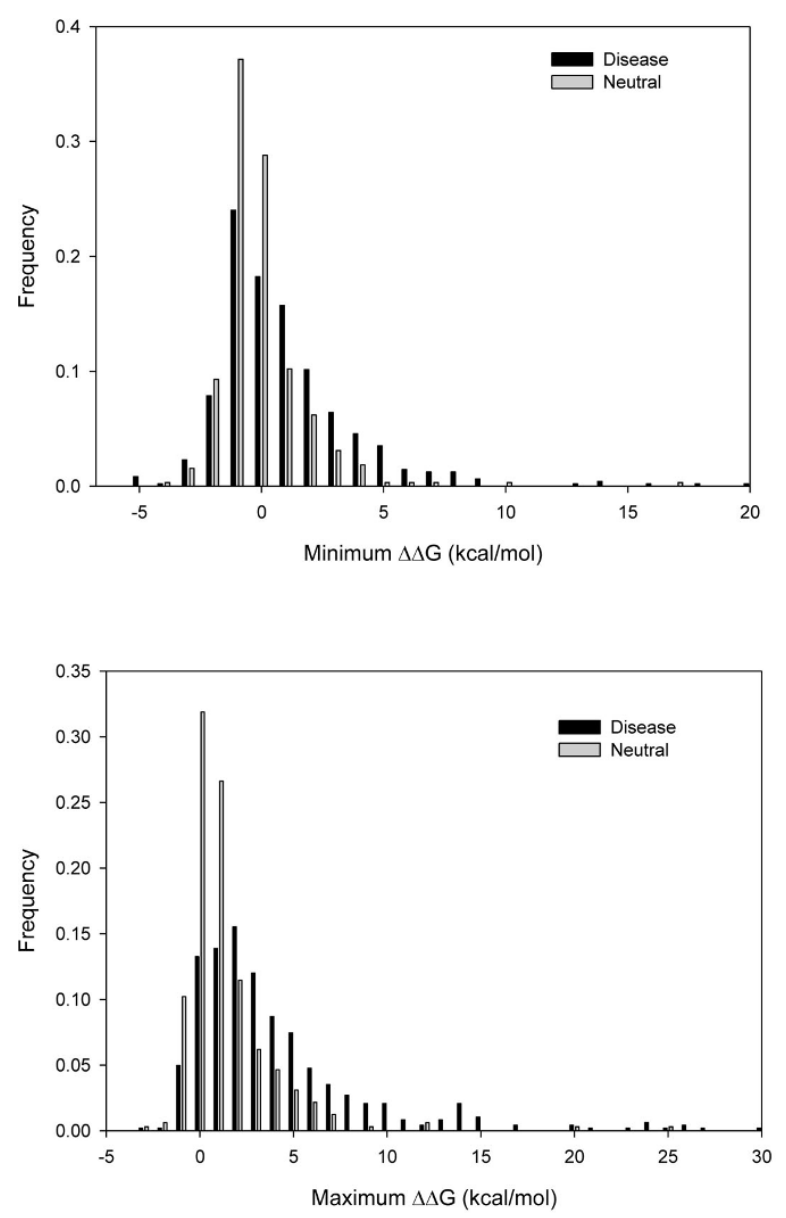

Figure 3 Distributions of maximum and minimum values of $\Delta \Delta G$ obtained for the different conformers for each protein in the dataset. Disease and neutral SASs are shown as separate distributions.

When each SAS is mapped into its corresponding protein, we found that in $35 \%$ of the cases there is at least one SAS that can be classified as neutral and stabilizing or neutral and destabilizing depending on the considered conformer. It is interesting to note that for this $35 \%$, the average of the maximum RMSD between conformers is $3.78 \AA$ compared to $2.38 \AA$ for proteins without ambiguous predicted stability changes. The discriminative threshold of $\Delta \Delta \mathrm{G}$ was set at $\pm 2 \mathrm{kcal} / \mathrm{mol}$, as it was previously discussed by Worth and coworkers (Worth, Preissner, and Blundell 2011). However, other works have defined different thresholds to classify the stability changes of a SAS [47-49]. When we analyzed the data distribution for $\Delta \Delta G$ values using a threshold of $\pm 1 \mathrm{kcal} / \mathrm{mol}$, the number of proteins with at least one ambiguous prediction was even higher (58\%) (these calculations can be done using the data and information included as additional file 1). Thus, the extension of conformational diversity measured by the maximum RMSD between conformers increases the variability of the stability prediction of SASs generating certain ambiguity in the prediction. It is noteworthy that this ambiguity is manifested in the same proportion respect to the total population of SASs in disease-associated SASs as in polymorphic SASs. That is, the proportion of neutral SASs with respect to the total number of SASs is similar to the proportion of neutral SASs with ambiguous prediction with respect to the total number of SASs with ambiguous predictions (almost 29\%). From this we can conclude that the uncertainty in the evaluation of the thermodynamic effect of a SAS equally affects neutral and disease related SASs.

In order to explore the effect of changes in ASA among conformers, we compared $\Delta(\Delta \Delta G)$ and $\Delta$ ASA values for disease and neutral SASs (Figure 5). It is interesting to note that at decreasing $\Delta$ ASA $(<0), \Delta(\Delta \Delta \mathrm{G})$ values indicate protein destabilization for disease related SASs $(\Delta(\Delta \Delta \mathrm{G})>2 \mathrm{kcal} / \mathrm{mol})$ and remain unaffected in the case of neutral SASs $(\Delta(\Delta \Delta \mathrm{G})<2 \mathrm{kcal} / \mathrm{mol})$. On the contrary, when positions become more exposed to solvent $(\Delta$ ASA $>0)$ the $\Delta \Delta G$ turns to stabilizing values. However, in this last condition, the neutral and disease related SASs present almost the same behavior. Most of the values between $\pm 50 \AA^{2}$ of $\triangle$ ASA (64\%) involve differences between exposed positions which explains the low variation in $\Delta(\Delta \Delta G)$ (approximately $\pm 2 \mathrm{kcal} / \mathrm{mol}$ ) as it is derived from the analysis of the distributions shown in Figure 5.

Summing up, all the observations indicate that there is a large spread of estimated $\Delta \Delta G$ values for the different conformers due to their structural differences. The spread and, eventually, the corresponding ambiguity in the prediction of $\Delta \Delta G$, can blur the correlation among $\Delta \Delta G$ values and the SAS type (disease related or neutral) when only one protein structure is used, as it is routinely the case.

\section{Prediction of disease related SASs using $\Delta \Delta \mathrm{G}$}

In order to explore how well the explicit consideration of conformational diversity could improve the estimation of disease related variants using stability measurements, we calculated the Mathews Correlation Coefficient (MCC) among $\Delta \Delta G$ computed values and the classification of the SASs as disease related or neutral. When conformational diversity is taken into account for each protein, a given SAS will result in a different number of $\Delta \Delta G$ estimated values (the number of values is equal to the number of conformers belonging to this protein). We first calculated MCC for all the data (14297 $\Delta \Delta G$ values for 119 proteins with an average number of conformers per protein of 8.6). Secondly, we characterized the change in the stability after a SAS using the minimum, maximum and average of the $\Delta \Delta$ Gs obtained from the set of corresponding conformers for a given protein and a given SAS. Finally, to contrast our hypothesis (that could be 

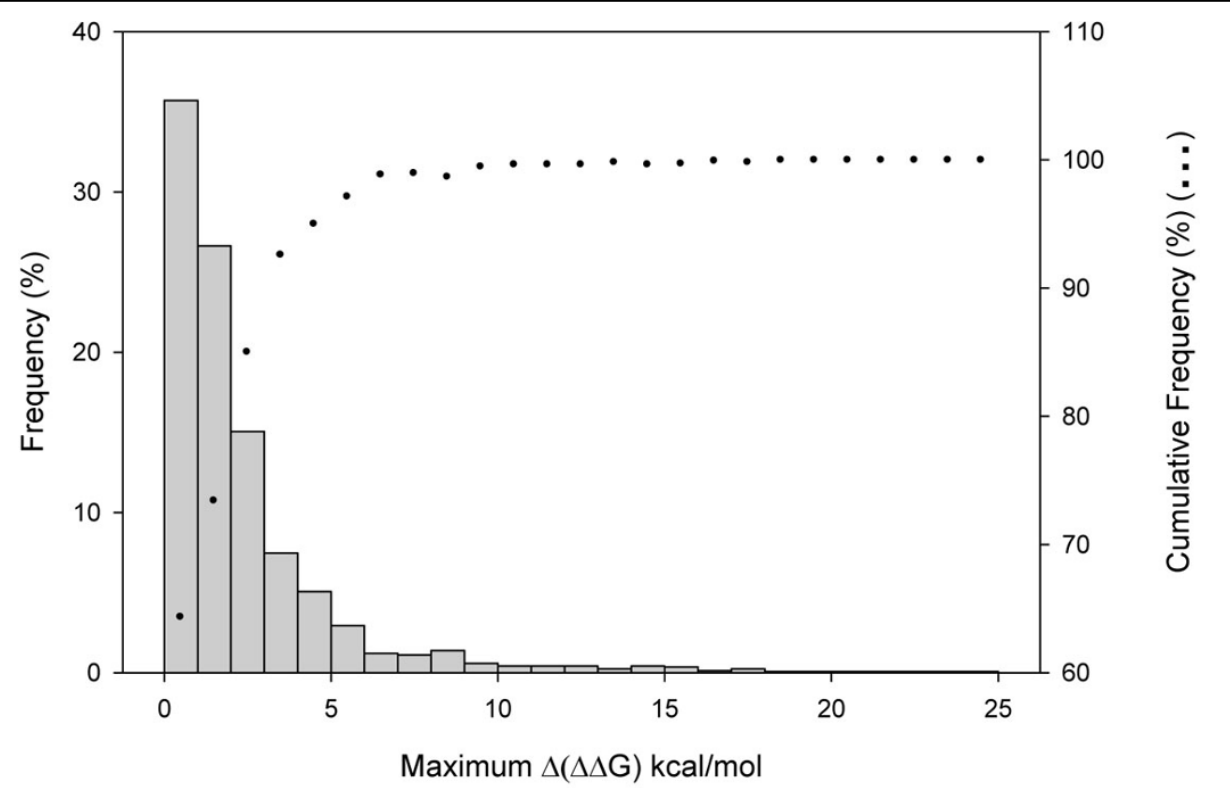

Figure 4 Distribution of maximum $\Delta(\Delta \Delta \mathrm{G})(\mathrm{kcal} / \mathrm{mol})$ for the 803 SASs studied. Bars represent the frequency and dots the cumulative frequency.

convenient to consider the conformational diversity) we also estimated MCC using a random selection of $\Delta \Delta G$ values derived from those obtained for each SAS in the set of corresponding conformers. The results, including the corresponding sensitivity, specificity and accuracy values are reported in Table 1 and indicate a significant difference between MCC corresponding to maximum values per conformer compared to random (P-value $=$ 0.02). Random gives an estimation of the performance of the use of the stability changes to predict disease related mutations using only one structure per protein in our

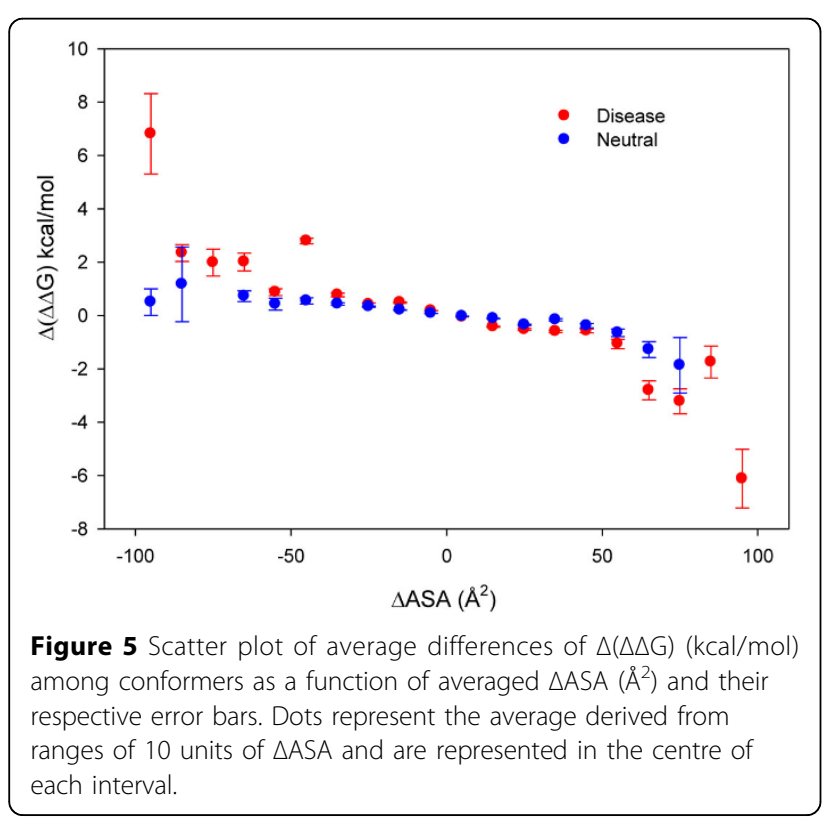

dataset. Our results for random selection of single $\Delta \Delta \mathrm{G}$ values agree with the performance reported in recent works [47]. The higher values of MCC using the maximum $\Delta \Delta G$ agree with the averaged maximum values for disease related $(4.62 \mathrm{kcal} / \mathrm{mol})$ and neutral SASs $(1.86 \mathrm{kcal} / \mathrm{mol})$ shown in Figure 3 . All the results are in agreement with previous work highlighting difficulties to discriminate disease related SASs on the basis of protein stability criteria [24].

As a final consideration, we observed that for each SAS in a given protein, and considering all conformer derived $\Delta \Delta G$ values, in 763 out of 803 SASs $(94.8 \%)$ there is at least one conformer whose $\Delta \Delta G$ value correlates perfectly with the disease or neutral phenotype (at a given/fixed $\Delta \Delta \mathrm{G}$ discriminative threshold). For some proteins in our

Table 1 Scoring the capability of discriminating among disease related and neutral SASs on different set of conformers.

\begin{tabular}{ccccc}
\hline & MCC & Accuracy & Specificity & Sensitivity \\
\hline Global* $^{\circ}$ & 0.19 & 0.54 & 0.76 & 0.44 \\
Minimum $^{\circ}$ & 0.23 & 0.54 & 0.85 & 0.34 \\
Maximum $^{\circ}$ & 0.36 & 0.68 & 0.69 & 0.68 \\
Average $^{\circ}$ & 0.25 & 0.62 & 0.80 & 0.5 \\
Random^^$^{\wedge}$ & 0.25 & 0.60 & 0.68 & 0.55 \\
\hline
\end{tabular}

*Global uses all data neglecting the correspondence between proteins and their conformers. ${ }^{\circ}$ Minimum, Maximum and Average characterize the values of $\Delta \Delta \mathrm{G}$ per SAS taking into account the conformers for each protein. $\wedge$ Random MCC was calculated as an average over 50 independent selections of a randomly taken one $\Delta \Delta \mathrm{G}$ value per SAS and per protein. In all the cases the threshold is $\pm 2 \mathrm{kcal} / \mathrm{mol}$. The difference between MCC values for Maximum and Random is significant at $\mathrm{P}$-value $=0.02$. For definition of the different scoring indexes see text. 
dataset, well populated with disease as well as with neutral SASs (25 proteins), we computed the conformer specific MCC to find the best conformer associated to disease or neutral phenotype. Interestingly most of these proteins show a different extent of variability of MCC values for the corresponding conformers. MCC values range from 0.67 to 1 for adenine phosphoribosyltransferase (P07741, with 4 conformers and 5 disease related and 1 neutral SASs), from 0.37 to 0.72 for fructose-biphosphate aldolase B2 (P05062, 2 conformers with 13 disease related and 3 neutral SASs) from 0.068 to 0.33 for the uroporphyrinogen decarboxylase (P06132, 7 conformers, 34 disease related and 6 neutral SASs) and from -0.04 to 0.27 for the transthyretin (P02766, 71 conformers, 74 disease related and 9 neutral SASs). In Figure 6 we show an example of how conformational diversity affects the estimation of $\Delta \Delta G$ and their correlation with disease. Cartoon representations for the two conformers considered for fructose-biphosphate aldolase B (P05062) are shown at the top; the central panel includes the values of $\Delta \Delta \mathrm{G}$ and at the bottom the corresponding MCC. Structural changes among conformers promote different local arrangements of variants resulting into different $\Delta \Delta G$ values. It is then expected that different conformers correlates in a different way with the occurrence of disease or neutral SASs. In order to obtain a further understanding on the role of the different conformers and the occurrence of disease or neutral SASs, we have mapped the occurrence of ligands in each conformer to detect bound and unbound states for each protein. We found that in $69 \%$ of the proteins the conformer with the maximum $\Delta \Delta G$ corresponds to the bound state of the protein. However this result should be taken with care. As explained in the Methods section we have detected the presence of ligands using Procognate database. In this procedure we just detected the presence of ligands that can be substrates, inhibitors, cofactors or allosteric effectors. Since different ligands change the population of conformers in different ways, the "bound" state could contain different conformers per protein. Considering the paucity of conformers available as compared to the protein universe, further work is necessary to completely elucidate their relevance to the disease related phenotype.

\section{Conclusions}

The elucidation of the effect that a single amino acid substitution (SAS) has in a specific phenotype is a central
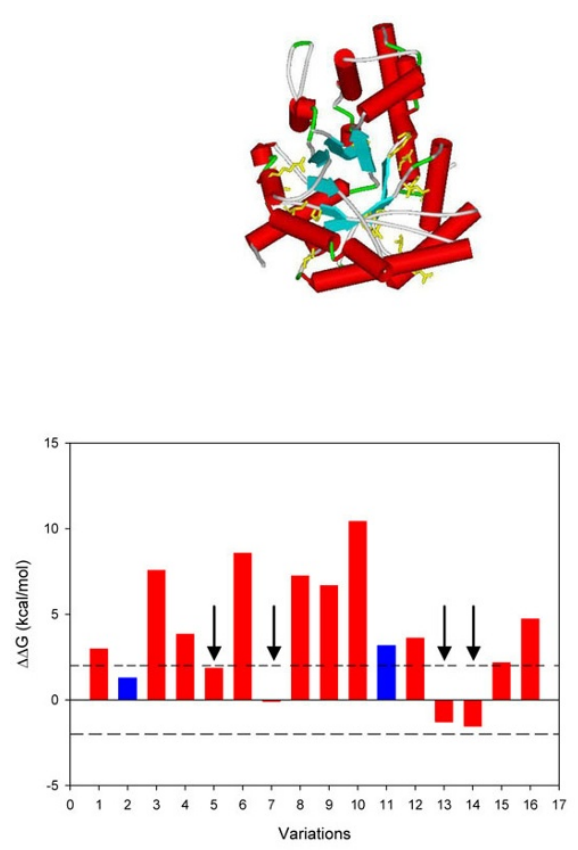

$\mathrm{MCC}=0.32$
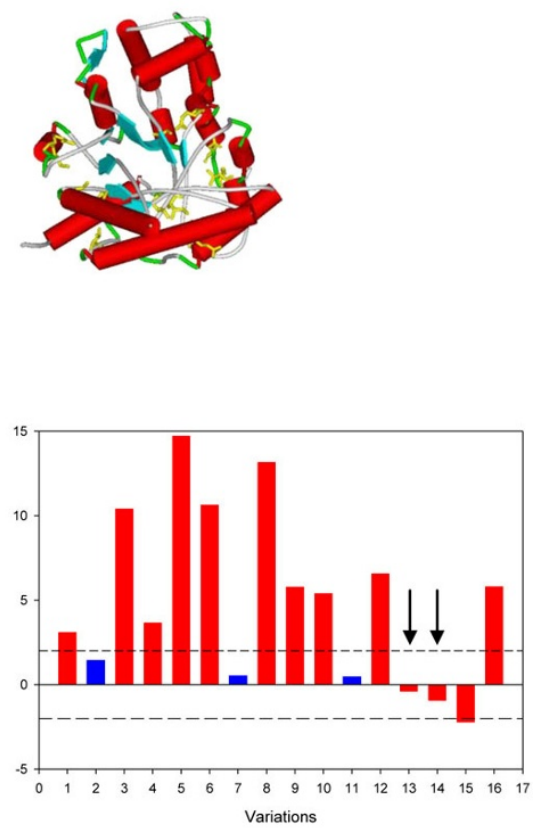

$\mathrm{MCC}=0.72$

Figure 6 Example showing how conformational diversity affects $\triangle \triangle G$ estimation and MCC values. The protein fructose-biphosphate aldolase B (P05062) is shown in the example. Two conformers were found for this protein with a RMSD =1.3 A. At the top of the figure there are cartoon representations of the two conformers with the mutated amino acids represented in stick (in yellow). Bars graphics represent $\Delta \Delta G$ values (red for disease associated mutations and blue for neutral mutations) and finally the corresponding MCC for each conformer. Black arrows indicate wrong predictions based in the reference interval of $\pm 2 \mathrm{kcal} / \mathrm{mol}$. As structural changes produce variations in $\Delta \Delta \mathrm{G}$ the different conformers have different MCC values. 
problem in different areas of research. Particularly, in protein computational biology it is very challenging to understand the mechanisms that lead to disease [50]. Estimation of protein stability and its perturbation after single SAS have played a key role to predict the effects of SASs $[1,6,11,51]$. Notwithstanding important advances in the area, the estimation of stability in proteins is still problematic and its correlation with disease or neutral variants highly depends on the dataset considered [24]. Here, we improved the correlation between the perturbation of protein stability and the classification of SASs as disease related or neutral. This improvement was obtained by considering maximum values of $\Delta \Delta \mathrm{G}$ computed taking into account conformational diversity. As the native state is not unique, the conservation of the biological activity of a protein relies on the different conformers of the ensemble. It has been found that the different conformers for a given protein constraints in different way the substitution pattern of proteins [52]. Then it is expected that the different conformers should have different robustness to SASs. Although the correlation is still far from being perfect, our results suggest that the effect of each SAS should be studied in all the protein conformers in order to obtain a better understanding of protein function perturbation and the disease origin. Our results also suggest that conformational diversity could add value to new computational tools for predicting SAS effects and play a key role to obtain a deeper understanding of the relationship among protein structure and function.

\section{Methods}

Data set collection

A list of proteins with disease and polymorphic SASs was extracted from http://www.uniprot.org/docs/humsavar. This list was linked with PCDB (http://pcdb.unq.edu.ar) database [43]. PCDB contains a collection of redundant protein structures obtained in different conditions (for example presence of ligands, change in oligomerization state, etc.). These structures can be considered as snapshots of protein dynamism, assumption validated by previous works that have proved the correspondence between structural deformations detected under different crystallographic conditions and conformational changes related to the flexibility of the native state $[39,40]$. The description of the conformational ensemble of the protein native state is limited to the information deposited in PCDB and PDB databases. The maximum RMSD (RMSDmax) between alpha carbon coordinates of the different conformers, calculated with MAMMOTH [53], is taken as a measure of the conformational diversity of the protein. From this cross linking, a dataset containing 119 proteins with different number of conformers was defined (8.6 conformers in average per protein, with a minimum of 2 and a maximum of 73). The length of all the structures in this dataset covers more than $70 \%$ of the length of the corresponding protein sequence. The dataset contains 803 SASs with 482 disease related and 323 neutral.

The presence of cognate ligands in each structure was determined using the Procognate database [54]. The cognate ligands deposited in this database are those involved in the biological function of the proteins. After this filtering we found that 35 proteins have bound/ unbound states in our dataset.

\section{Stability and structural measurements}

For each SAS and for each protein and conformation, we estimated $\Delta \Delta G$ values using the program FoldX [44]. FoldX uses an empirical potential calibrated to fit in vitro $\Delta \Delta G$ values. Area exposed to solvent was calculated with Naccess program (http://www.bioinf.manchester.ac.uk/naccess/).

\section{Statistical analysis}

To study the performance to predict disease or neutral variants using estimated $\Delta \Delta G$ values, we calculated the accuracy, specificity, sensitivity and also the Matthew's correlation coefficient (MCC). The equations used were:

$$
\begin{aligned}
& \text { Accuracy }=\frac{t p+t n}{t p+t n+f p+f n} \\
& \text { Specificity }=\frac{t n}{t n+f p} \\
& \text { Sensitivity }=\frac{t p}{t p+f n} \\
& M c c=\frac{t p t n-f n f p}{\sqrt{(t p+f n)(t p+f p)(t n+f n)(t n+f p)}}
\end{aligned}
$$

A value of $\mathrm{MCC}=1$ defines the best possible prediction, while a value of $\mathrm{MCC}=-1$ indicates the worst possible prediction. A value of $\mathrm{MCC}=0$ corresponds to predictions made by chance.

\section{Additional material}

Additional file 1: List of SASs mapped on the corresponding structures. List of proteins, structures and corresponding conformers.

\section{Acknowledgements}

RC thanks the following grants: PRIN 2009 project 009WXT45Y (Italian Ministry for University and Research: MIUR), COST BMBS Action TD1101 
(European Union RTD Framework Programme), and PON project PON01_02249 (Italian Ministry for University and Research: MIUR). MSF thanks Erasmus Mundus Action 2 Lot 13a - EADIC II proyect, funded by the European Commission and coordinated by the University of Bologna for the research fellowship. GP and MSF thank the following grants: PIP CONICET (112-200801-02849) and UNQ (1004/11). EJ has a Type II fellowship from CONICET.

This article has been published as part of BMC Genomics Volume 13 Supplement 4, 2012: SNP-SIG 2011: Identification and annotation of SNPs in the context of structure, function and disease. The full contents of the supplement are available online at http://www.biomedcentral.com/ bmcgenomics/supplements/13/S4.

\section{Author details}

'Departamento de Ciencia y Tecnología, Universidad Nacional de Quilmes, Buenos Aires, Argentina. ${ }^{2}$ Biocomputing Group, Department of Biology, University of Bologna, Italy. ${ }^{3}$ Biocomputing Group, Department of Computer Science, University of Bologna, Italy.

\section{Competing interests}

The authors declare that they have no competing interests.

\section{Published: 18 June 2012}

\section{References}

1. Wang Z, Moult J: SNPs, protein structure, and disease. Hum Mutat 2001, 17(4):263-270.

2. Lofgren M, Banerjee R: Loss of allostery and coenzyme B12 delivery by a pathogenic mutation in adenosyltransferase. Biochemistry 2011, 50(25):5790-5798.

3. Shah GN, Bonapace G, Hu PY, Strisciuglio P, Sly WS: Carbonic anhydrase II deficiency syndrome (osteopetrosis with renal tubular acidosis and brain calcification): novel mutations in CA2 identified by direct sequencing expand the opportunity for genotype-phenotype correlation. Hum Mutat 2004, 24(3):272.

4. Orosz F, Olah J, Ovadi J: Triosephosphate isomerase deficiency: new insights into an enigmatic disease. Biochim Biophys Acta 2009, 1792(12):1168-1174

5. Almeida-Souza L, Goethals S, de Winter V, Dierick I, Gallardo R, Van Durme J, Irobi J, Gettemans J, Rousseau F, Schymkowitz J, et al: Increased monomerization of mutant HSPB1 leads to protein hyperactivity in Charcot-Marie-Tooth neuropathy. J Biol Chem 2010, 285(17):12778-12786.

6. Yue $P, L i Z$, Moult J: Loss of protein structure stability as a major causative factor in monogenic disease. J Mol Biol 2005, 353(2):459-473.

7. Ling SC, Albuquerque CP, Han JS, Lagier-Tourenne C, Tokunaga S, Zhou H, Cleveland DW: ALS-associated mutations in TDP-43 increase its stability and promote TDP-43 complexes with FUS/TLS. Proc Natl Acad Sci U S A 2010, 107(30):13318-13323.

8. Seidle HF, Bieganowski P, Brenner C: Disease-associated mutations inactivate AMP-lysine hydrolase activity of Aprataxin. J Biol Chem 2005, 280(22):20927-20931.

9. Alonso Adel C, Mederlyova A, Novak M, Grundke-lqbal I, Iqbal K: Promotion of hyperphosphorylation by frontotemporal dementia tau mutations. J Biol Chem 2004, 279(33):34873-34881.

10. Dobson $C M$ : The structural basis of protein folding and its links with human disease. Philos Trans R Soc Lond B Biol Sci 2001, 356(1406):133-145.

11. Casadio R, Vassura M, Tiwari S, Fariselli $P$, Luigi Martelli P: Correlating disease-related mutations to their effect on protein stability: a largescale analysis of the human proteome. Hum Mutat 2011, 32(10):1161-1170

12. Gromiha MM, An J, Kono H, Oobatake M, Uedaira H, Sarai A: ProTherm: Thermodynamic Database for Proteins and Mutants. Nucleic Acids Res 1999, 27(1):286-288.

13. Bash PA, Singh UC, Langridge R, Kollman PA: Free energy calculations by computer simulation. Science 1987, 236(4801):564-568.

14. Lee C: Predicting protein mutant energetics by self-consistent ensemble optimization. J Mol Biol 1994, 236(3):918-939.

15. Lee C, Levitt M: Accurate prediction of the stability and activity effects of site-directed mutagenesis on a protein core. Nature 1991, 352(6334):448-451.
16. Koehl P, Delarue M: Polar and nonpolar atomic environments in the protein core: implications for folding and binding. Proteins 1994, 20(3):264-278.

17. Munoz V, Serrano L: Intrinsic secondary structure propensities of the amino acids, using statistical phi-psi matrices: comparison with experimental scales. Proteins 1994, 20(4):301-311.

18. Miyazawa $\mathrm{S}$, Jernigan RL: Protein stability for single substitution mutants and the extent of local compactness in the denatured state. Protein Eng 1994, 7(10):1209-1220.

19. Sippl MJ: Knowledge-based potentials for proteins. Curr Opin Struct Biol 1995, 5(2):229-235.

20. Khan S, Vihinen M: Performance of protein stability predictors. Hum Mutat 2010, 31(6):675-684.

21. Yue P, Melamud E, Moult J: SNPs3D: candidate gene and SNP selection for association studies. BMC Bioinformatics 2006, 7:166.

22. Schymkowitz J, Borg J, Stricher F, Nys R, Rousseau F, Serrano L: The FoldX web server: an online force field. Nucleic Acids Res 2005, 33(Web Server issue):W382-388

23. Dehouck Y, Kwasigroch JM, Gilis D, Rooman M: PoPMuSiC 2.1 : a web server for the estimation of protein stability changes upon mutation and sequence optimality. BMC Bioinformatics 12(1):151.

24. Reumers J, Schymkowitz J, Rousseau F: Using structural bioinformatics to investigate the impact of non synonymous SNPs and disease mutations: scope and limitations. BMC Bioinformatics 2009, 10(Suppl 8):S9.

25. James LC, Tawfik DS: Conformational diversity and protein evolution-a 60-year-old hypothesis revisited. Trends Biochem Sci 2003, 28(7):361-368.

26. Lange OF, Lakomek NA, Fares C, Schroder GF, Walter KF, Becker S, Meiler J, Grubmuller H, Griesinger C, de Groot BL: Recognition dynamics up to microseconds revealed from an RDC-derived ubiquitin ensemble in solution. Science 2008, 320(5882):1471-1475.

27. Tsai CD, Ma B, Kumar S, Wolfson H, Nussinov R: Protein folding: binding of conformationally fluctuating building blocks via population selection. Crit Rev Biochem Mol Biol 2001, 36(5):399-433.

28. del Sol A, Tsai CJ, Ma B, Nussinov R: The origin of allosteric functional modulation: multiple pre-existing pathways. Structure 2009, 17(8):1042-1050

29. Hilser VJ: Biochemistry. An ensemble view of allostery. Science 2010, 327(5966):653-654

30. Karplus M, Kuriyan J: Molecular dynamics and protein function. Proc Natl Acad Sci U S A 2005, 102(19):6679-6685.

31. Khersonsky O, Roodveldt C, Tawfik DS: Enzyme promiscuity: evolutionary and mechanistic aspects. Curr Opin Chem Biol 2006, 10(5):498-508.

32. James LC, Roversi P, Tawfik DS: Antibody multispecificity mediated by conformational diversity. Science 2003, 299(5611):1362-1367.

33. Boehr DD, McElheny D, Dyson HJ, Wright PE: The dynamic energy landscape of dihydrofolate reductase catalysis. Science 2006, 313(5793):1638-1642.

34. Smock RG, Gierasch LM: Sending signals dynamically. Science 2009, 324(5924):198-203.

35. Yogurtcu ON, Erdemli SB, Nussinov R, Turkay M, Keskin O: Restricted mobility of conserved residues in protein-protein interfaces in molecular simulations. Biophys J 2008, 94(9):3475-3485.

36. Tokuriki N, Tawfik DS: Protein dynamism and evolvability. Science 2009 324(5924):203-207.

37. Ma B, Shatsky M, Wolfson HJ, Nussinov R: Multiple diverse ligands binding at a single protein site: a matter of pre-existing populations. Protein $\mathrm{Sci}$ 2002, 11(2):184-197.

38. Gunasekaran $\mathrm{K}, \mathrm{Ma} \mathrm{B}$, Nussinov R: Is allostery an intrinsic property of all dynamic proteins? Proteins 2004, 57(3):433-443.

39. Best RB, Lindorff-Larsen $\mathrm{K}$, DePristo MA, Vendruscolo M: Relation between native ensembles and experimental structures of proteins. Proc Natl Acad Sci U S A 2006, 103(29):10901-10906.

40. Zoete V, Michielin O, Karplus M: Relation between sequence and structure of HIV-1 protease inhibitor complexes: a model system for the analysis of protein flexibility. J Mol Biol 2002, 315(1):21-52.

41. Burra PV, Zhang Y, Godzik A, Stec B: Global distribution of conformational states derived from redundant models in the PDB points to nonuniqueness of the protein structure. Proc Natl Acad Sci U S A 2009, 106(26):10505-10510 
42. Berman HM, Westbrook J, Feng Z, Gilliland G, Bhat TN, Weissig H, Shindyalov IN, Bourne PE: The Protein Data Bank. Nucleic Acids Res 2000, 28(1):235-242.

43. Juritz J, Fernandez-Alberti S, Parisi G: PCDB: A database of proteins with conformational diversity. Nucleic Acids Res 2010, 39:D475-9.

44. Guerois R, Nielsen JE, Serrano L: Predicting changes in the stability of proteins and protein complexes: a study of more than 1000 mutations. $J$ Mol Biol 2002, 320(2):369-387.

45. Kumar S, Ma B, Tsai CJ, Sinha N, Nussinov R: Folding and binding cascades: dynamic landscapes and population shifts. Protein Sci 2000, 9(1):10-19.

46. Tokuriki N, Stricher F, Schymkowitz J, Serrano L, Tawfik DS: The stability effects of protein mutations appear to be universally distributed. $J \mathrm{Mol}$ Biol 2007, 369(5):1318-1332.

47. Worth $C L$, Preissner R, Blundell TL: SDM-a server for predicting effects of mutations on protein stability and malfunction. Nucleic Acids Res 2011.

48. Calloni G, Zoffoli S, Stefani M, Dobson CM, Chiti F: Investigating the effects of mutations on protein aggregation in the cell. J Biol Chem 2005, 280(11):10607-10613.

49. Tokuriki N, Tawfik DS: Stability effects of mutations and protein evolvability. Curr Opin Struct Biol 2009, 19(5):596-604.

50. Thusberg J, Vihinen M: Pathogenic or not? And if so, then how? Studying the effects of missense mutations using bioinformatics methods. Hum Mutat 2009, 30(5):703-714.

51. Worth CL, Bickerton GR, Schreyer A, Forman JR, Cheng TM, Lee S, Gong S, Burke DF, Blundell TL: A structural bioinformatics approach to the analysis of nonsynonymous single nucleotide polymorphisms (nsSNPs) and their relation to disease. J Bioinform Comput Biol 2007, 5(6):1297-1318.

52. Juritz J, Palopoli N, Fornasari M, Fernandez-Alberti S, Parisi G: Protein conformational diversity modulates protein divergence. Accepted Mol Biol Evol 20122012.

53. Ortiz AR, Strauss CE, Olmea O: MAMMOTH (matching molecular models obtained from theory): an automated method for model comparison. Protein Sci 2002, 11(11):2606-2621.

54. Bashton M, Nobeli I, Thornton JM: PROCOGNATE: a cognate ligand domain mapping for enzymes. Nucleic Acids Res 2008, 36(Database issue): D618-622.

doi:10.1186/1471-2164-13-S4-S5

Cite this article as: Juritz et al:: On the effect of protein conformation diversity in discriminating among neutral and disease related single amino acid substitutions. BMC Genomics 2012 13(Suppl 4):S5.

\section{Submit your next manuscript to BioMed Central and take full advantage of:}

- Convenient online submission

- Thorough peer review

- No space constraints or color figure charges

- Immediate publication on acceptance

- Inclusion in PubMed, CAS, Scopus and Google Scholar

- Research which is freely available for redistribution

Submit your manuscript at www.biomedcentral.com/submit
Biomed Central 